\title{
Learning to ignore: Acquisition of sustained attentional suppression
}

\author{
Matthew L. Dixon, Justin Ruppel, and Jay Pratt \\ University of Toronto, Toronto, Ontario, Canada \\ AND \\ Eve De Rosa \\ University of Toronto and Rotman Research Institute, Toronto, Ontario, Canada
}

\begin{abstract}
We examined whether the selection mechanisms committed to the suppression of ignored stimuli can be modified by experience to produce a sustained, rather than transient, change in behavior. Subjects repeatedly ignored the shape of stimuli, while attending to their color. On subsequent attention to shape, there was a robust and sustained decrement in performance that was selective to when shape was ignored across multiple-colortarget contexts, relative to a single-color-target context. Thus, amount of time ignored was not sufficient to induce a sustained performance decrement. Moreover, in this group, individual differences in initial color target selection were associated with the subsequent performance decrement when attending to previously ignored stimuli. Accompanying this sustained decrement in performance was a transfer in the locus of suppression from an exemplar (e.g., a circle) to a feature (i.e., shape) level of representation. These data suggest that learning can influence attentional selection by sustained attentional suppression of ignored stimuli.
\end{abstract}

It is well appreciated that, despite constant competition for attention from an overabundance of irrelevant information, we are remarkably capable of selectively attending to behaviorally relevant information. The "biased competition" model of attention (Desimone \& Duncan, 1995) proposes that attentional mechanisms resolve this competition by enhancing and suppressing the representations of behaviorally relevant and irrelevant stimuli, respectively (see also Navalpakkam \& Itti, 2007; Serences $\&$ Yantis, 2006). But it is rare that we interact with complex environments without any expectations; rather, we acquire expectations and redundancies about our environment with experience. Thus, learning likely also plays an important role in our ability to select behaviorally relevant information and ignore information that is consistently irrelevant. Indeed, Desimone and Duncan postulated that learning may play an important role in modulating stimulus competition for attention, though little empirical work has sought to test this hypothesis.

It is known that perceptual learning can occur for both task-relevant attended (Dosher \& Lu, 1998; Op de Beeck, Baker, DiCarlo, \& Kanwisher, 2006) and task-irrelevant unattended stimuli (Seitz \& Watanabe, 2003; Watanabe, Náñez, \& Sasaki, 2001). One means by which these effects could be attained is through the altered tuning of attentional filters. Recent evidence has shown that experience can directly modulate attentional processes-specifically, enhancing target detection during visual search (Chun \& Jiang, 1998, 1999). It currently remains unknown, however, to what extent learning may enhance performance by tuning attentional filters to suppress ignored information. This concept of experience modulating the attentional mechanisms that suppress ignored sensory input has received support from electrophysiological data in nonhuman primates (e.g., Ipata, Gee, Gottlieb, Bisley, \& Goldberg, 2006); therefore, we have chosen to use the term suppression.

Suppression mechanisms should be more influenced by the redundancy of the ignored status of stimuli and less influenced by the mere amount of time that stimuli are ignored (Mackintosh, 1975). Accordingly, a stimulus that is consistently task irrelevant across different contexts may be suppressed in a more sustained manner to provide a reduction in the perceptual "noise" that competes for attention. A central aspect of learning is also the ability to generalize from experience, since it allows organisms to predict how to process information efficiently for behavior in novel situations (Poggio \& Bizzi, 2004). Although generalization has been thoroughly examined in the context of perceptual learning (Fahle, 2005), it remains unknown whether mechanisms of attentional suppression are sensitive to, and generalize from, experience.

In the present study, we examined whether experience can (1) modify the strength and duration of suppression of exemplars (e.g., a circle) from an ignored stimulus dimension and (2) tune attentional filters so that suppression will generalize beyond the specific ignored exemplars to encompass the feature category to which they belong (i.e., shape). To address these questions, we designed a simple simultaneous

E.De Rosa, derosa@psych.utoronto.ca 


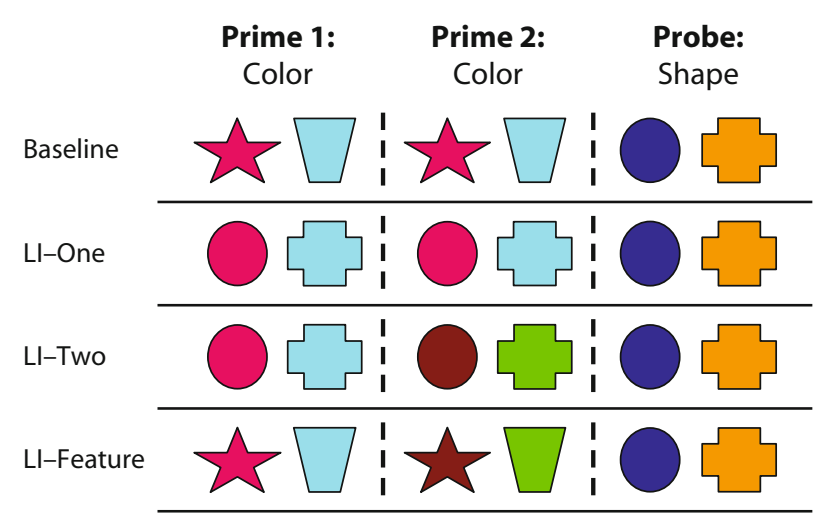

Figure 1. A simplified depiction of target colored shapes for each block for the four object discrimination tasks: (1) baseline, (2) learning to ignore-one context ( $\mathrm{LI}-$-one), (3) learning to ignore-two contexts (LI-two), and (4) learning to ignore-feature (LI-feature).

object discrimination paradigm during which subjects selected prespecified target objects. During two prime blocks, efficient target selection required attending to the color of the objects and ignoring their shape, and during a subsequent probe block, efficient target selection required attending to the shape and ignoring the color of the objects. According to the biased competition model (Desimone \& Duncan, 1995), if shape information is suppressed during prime blocks, then it should be less able to compete with color information for attention during the probe block, and consequently, a performance decrement should be evident. Importantly, we assessed suppression in terms of a decrement over and above a basic task-switch cost associated with reorienting attention from one feature dimension to the other. Although we assume an attentional suppression mechanism to account for the potential slowed response to previously ignored stimuli, it should be noted that a plausible episodic retrieval mechanism also exists - that is, the performance decrement may reflect the retrieval of previous episodes associated with the same stimulus (see Tipper, 2001).

To index attentional suppression independently of competition, we designed the tasks so that (1) novel distractor colors were present during the probe block to eliminate stimulus competition and (2) subjects were never required to respond to the shape dimension until the probe block, to eliminate response competition from the prime block. Both of these design features allowed us to assess the representation of the ignored dimension in a relatively independent manner. This paradigm also allows for the potential accrual of suppression to those features across many prime trials. In contrast to previous work, we monitored performance across multiple probe trials to assess the magnitude as well as the resolution of the cost for attending to previously ignored stimuli.

\section{EXPERIMENT 1A}

\section{Method}

Subjects. Eighty-one University of Toronto undergraduate students $\left(M_{\text {age }}=21.3, S D=4.81\right)$ received course credit or financial payment for their participation in these studies.
Simultaneous object discrimination paradigm. The present study used four simultaneous object discrimination tasks (Figure 1). Subjects were randomly assigned to one of the four tasks: baseline, learning to ignore-one context (LI-one), learning to ignore-two contexts (LI-two), or learning to ignore-feature (LI-feature). The four tasks were designed to produce identical cognitive and response demands and to differ only in the prior exposure history of the previously ignored distractors during probe trials.

In each task, subjects performed three blocks of trials: two prime blocks followed by a probe block. At the beginning of each block, subjects were presented with an array of four target objects that were always a combination of two colors and two shapes (Figure 2); each block consisted of 96 trials. In each trial, two colored objects $\left(16.5^{\circ}\right.$ high $\times 13.5^{\circ}$ wide $)$ were simultaneously presented for $700 \mathrm{msec}$, followed by a 220-msec fixation cross; one object was always a target and the other was always a distractor. Subjects selected the side of the screen (right or left) on which the target was located using one of two prespecified keys. Stimulus identity and location were counterbalanced and presented pseudorandomly across trials. Auditory feedback was presented immediately after each response.

During prime trials, target and distractor stimuli had the same shapes, but different colors. This encouraged subjects to attend to color and ignore shape information to accurately identify the targets. In contrast, during probe trials, target and distractor stimuli had the same colors, but different shapes, encouraging subjects to attend to shape and ignore color information to identify these targets.

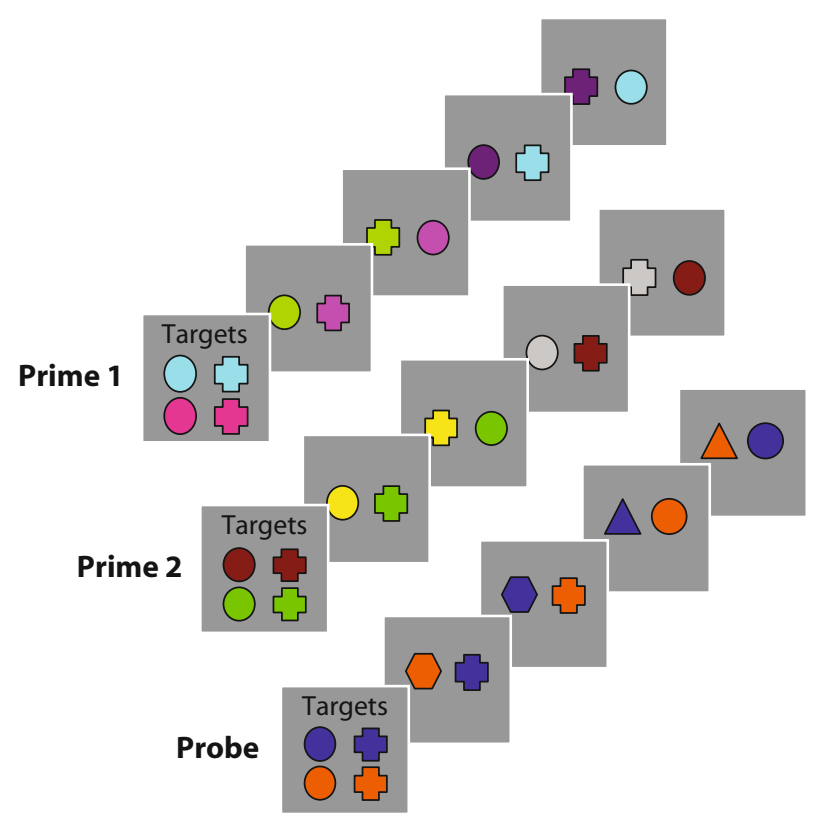

Figure 2. Depiction of each trial type for each block of the learning to ignore-two contexts task. Subjects indicate on which side the target appears. For simplicity, all targets appear on the right side of the display, although target location was counterbalanced and pseudorandomly presented during the experiment. (A) Prime 1 block. Subjects learned to attend to stimulus color to discriminate pink targets from olive distractors and aqua targets from purple distractors, while ignoring the uninformative shape information. (B) Prime 2 block. Subjects learned to attend to color to discriminate green targets from yellow distractors and brown targets from gray distractors, while ignoring the uninformative shape information. (C) Probe block. Subjects learned to attend to the stimulus shape to discriminate cross targets from triangle distractors and circle targets from hexagon distractors. 
Attentional manipulation. The target objects were always a combination of two colors and two shapes (see Figure 2). During prime trials, target and distractor stimuli had the same shapes, but different colors. This encouraged subjects to attend to color and ignore shape information to identify the targets quickly. In contrast, during probe trials, target and distractor stimuli had the same colors, but different shapes, encouraging subjects to attend to shape and ignore color information to identify the targets. Importantly, we did not instruct subjects to attend to color or shape; they naturally adopted this strategy through performance feedback. Nearly all subjects reported using this strategy during postexperiment debriefing.

The four tasks differed in the particular colors that were attended and shapes that were ignored during the prime block, but were identical in the shapes attended during the probe block (Figure 1). We assessed attentional suppression as the "performance cost" when subjects had to reorient attention away from the color dimension to the previously ignored shape dimension during probe trials. Thus, by examining the relative performance cost during the probe block of each task, we were able to examine how subjects' ability to attend to identical shapes was altered by their particular prime experience. Importantly, we used novel colors during the probe to minimize the influence of past color associations to allow probe performance to largely reflect subjects' past experience with shape information alone. Neither color nor shape information was associated with a fixed response at any time; this eliminated the requirement to inhibit a competing prepotent response. The four tasks were:

Baseline. In the baseline task, the attended colors and ignored shapes remained constant across both prime blocks. Then, during the probe block, subjects attended to novel shapes, not previously ignored shapes, as in the remaining tasks. Thus, comparing the performance cost of baseline relative to the remaining tasks allowed us to (1) account for any natural asymmetry that may exist in attending to color or shape and (2) also assess, above and beyond that potential asymmetry, the performance cost for reorienting attention to previously ignored shape information; this assessment could serve as an index of attentional suppression.

Learning to ignore-one context. The LI-one task examined suppression of particular shape exemplars strictly as a function of the amount of time ignored. Throughout the prime blocks, the attended colors and ignored shapes remained constant. In contrast to the baseline task, subjects in this task attended to the previously ignored shape exemplars during the probe block, in the presence of novel colors. A probe performance decrement over and above the baseline cost would reveal a suppression of the previously ignored shape exemplars.

Learning to ignore-two contexts. The LI-two task examined whether suppression would be greater if the task irrelevance of the shape exemplars was reinforced across two-distinct-color-target contexts. This task is identical to LI-one, with the exception that a new set of target colors was introduced during Prime Block 2 . A greater probe performance decrement relative to both baseline and LI-one would reveal an experience-dependent modulation of presumed attentional suppression.

Learning to ignore-feature. The LI-feature task was designed to examine whether suppression would generalize with experience. In this task, similar to LI-two, identical shape exemplars were ignored across two different sets of target colors during prime blocks. However, in this case, subjects attended to novel shapes, rather than to the exact previously ignored shapes, during the probe. If the probe performance decrement when attending to novel shapes is greater than the baseline cost and similar to the LI-two cost, then this would imply that suppression has generalized beyond the particular shape exemplars that were ignored.

Practice. All subjects practiced four attend-color blocks and then four attend-shape blocks, for a total of 224 practice trials. The first blocks for each dimension were 16 trials, and stimuli were presented until a response was made (i.e., they were self-paced). The remaining three blocks were 32 trials in length.

Statistical analyses. Prior work (Tipper, 1985) indicates that suppression of task-irrelevant information is contingent upon adequate selection of target information. We therefore adopted a performance criterion of at least $75 \%$ accuracy across the first prime block for inclusion of a subject's data in the analyses. This led to the removal of 1 subject from baseline $(n=19), 4$ from LI-one $(n=18), 1$ from LI-two $(n=19)$, and 1 from LI-feature $(n=18)$.

\section{Results}

To explore performance across time, we divided the 96trial prime and probe blocks into 24-trial bins (or quartiles); then for each subject, we computed median reaction time (RT) for correct responses and mean accuracy for each quartile. The performance cost was computed by taking scores at each quartile of the probe block and subtracting scores for the last quartile of the temporally preceding Prime Block 2, where performance was most stable. RT and accuracy were submitted to a 4 (task) $\times 4$ (quartile) mixed repeated measures ANOVA, with task as a betweengroup variable and quartile as a within-group variable. Planned pairwise comparisons were performed on the main effect of task, and when appropriate, data at specific quartiles were analyzed with independent samples $t$ tests. Additionally, when appropriate, Greenhouse-Geisser adjustments to the degrees of freedom were performed and corrected $p$ values were reported. Cohen's $d$ was used as an estimate of effect size.

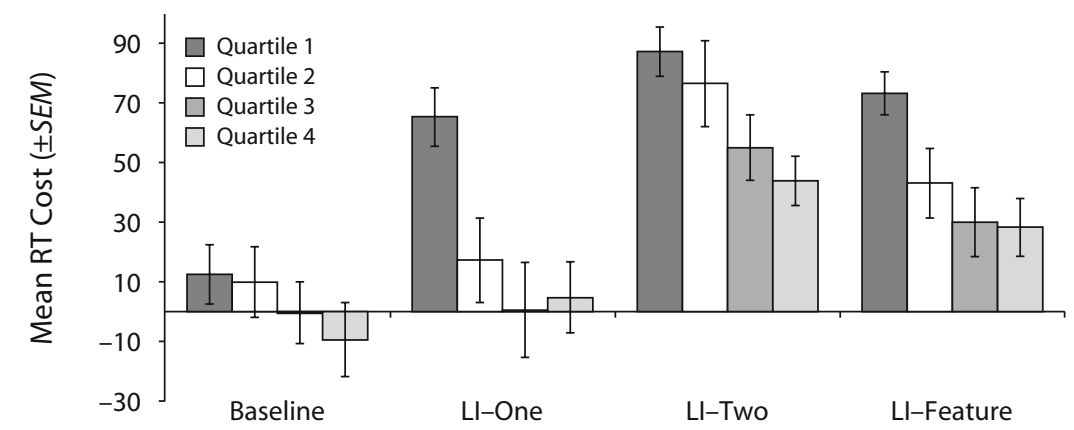

Figure 3. The mean reaction time (RT) performance cost in milliseconds $( \pm S E M)$ for each quartile of the probe block for each task. Each quartile consists of 24 trials. 
Accuracy was very high and probe performance did not vary as a function of task, or a task $\times$ quartile interaction $(p>.05)$.

Figure 3 depicts the mean RT performance cost at each probe quartile as a function of the task performed. The probe performance decrement varied significantly as a function of task $[F(3,70)=7.56, p<.001]$, quartile $[F(2.67,187.13)=36.60, p<.001]$, and task $\times$ quartile $[F(8.02,187.13)=2.65, p=.009]$. First we measured performance associated with switching attention from color to shape, to provide a pure measure of dimensional task switching. The baseline cost was minimal and did not persist. Thus, this behavior will serve as an index of the basic requirement to reorient attention from one dimension to the other. Additionally, it demonstrated that there was not a greater tendency to direct attention to color information and that there was little interference from response competition during the probe.

In the LI-one task, subjects attended to the exact shapes during the probe block that they ignored in the prime. The overall performance cost (collapsed across all quartiles) for baseline and LI-one did not differ $[F(1,35)=1.88, p=.18]$. However, the LI-one cost was significantly greater than the baseline cost during the first quartile alone $[t(35)=3.42, p=.002, d=1.13]$; (Quartile 1 cost: $M_{\mathrm{LI}-\mathrm{one}}=65.14 \mathrm{msec}, S D=50.4$; $\left.M_{\text {baseline }}=12.42 \mathrm{msec}, S D=43.2\right)$. Color information was attended and shape information ignored for the same amount of time in the baseline and LI-one tasks; all that differed was whether subjects attended to novel or previously ignored shapes during the probe. Thus, LI-one performance reflects the suppression of the previously ignored shape exemplars. Interestingly, the transient nature of the LI-one decrement suggests that if a stimulus is ignored - even repeatedly - in only one context during the prime, suppression mechanisms are rapidly disengaged during the probe block.

In LI-two, where shape exemplars were consistently ignored across two-distinct-color-target contexts, subjects evidenced a substantial performance decrement (Quartile 1 cost: $M_{\text {LI-two }}=87.08 \mathrm{msec}, S D=44.78$ ) that persisted throughout the entire 96-trial probe block. This result contrasts with transient performance decrements in LI-one and baseline. Comparing the average decrement across the entire probe block, the LI-two condition significantly exceeded baseline and LI-one $[F(1,36)=$ $22.42, p<.001, d=1.54$, and $F(1,35)=7.25, p=.011$, $d=0.89$, respectively]. Thus, experience with the ignored shape exemplars across more than one color target context led to a more robust and sustained suppression, even though the exemplars were ignored for the same number of trials as in the LI-one and baseline tasks.

The LI-feature condition was similar to the baseline condition, in that no colors or shapes at probe were common with the prime block, but it differed from the baseline condition in that prime shapes were ignored in twocolor contexts. When responding to novel shapes in the LI-feature condition, there was a substantial performance decrement (Quartile 1 cost: $M_{\text {LI-feature }}=73.08 \mathrm{msec}$,
$S D=52.42)$ comparable with LI-two $(p>.05)$ that persisted for the duration of the probe block. The overall performance decrement was substantially larger than baseline $[F(1,35)=12.50, p=.001, d=1.16]$. These findings demonstrate that learning to ignore shapes in multiple contexts led to a broadening of distractor suppression so that it generalized to novel shapes.

Target processing efficiency and attentional suppression. One important function of attentional suppression of ignored information is that by decreasing attention to ignored information (noise) one can enhance processing efficiency of behaviorally relevant information (signal), thus altering the task signal-to-noise ratio with experience. To examine this, we determined whether individuals who revealed the greatest performance decrement at probe demonstrated the greatest efficiency in prime target selection by correlating a prime difference score (Prime 2 RT - Prime 1 RT) with our performance cost for reorienting attention to the ignored shape dimension at probe. There was no relationship when shape exemplars were ignored in only a one-color-target context - that is, baseline and LI-one $(r=.16, p=.33)$. But there was a significant negative relationship when shape exemplars were ignored in two-color-target contexts - that is, LI-two and LI-feature $(r=-.42, p=.01)$ - demonstrating that individuals with the most robust probe performance decrement were better at target selection during the prime. Performance during Prime 2 did not vary as a function of task $(F<1)$; that is, performance improved during Prime 2 in all tasks. This improvement was selectively predictive of the probe performance decrement when the task irrelevance of the ignored shape dimension was reinforced; this reveals a direct relationship between experience, presumed attentional suppression, and target selection efficiency.

\section{EXPERIMENT 1B}

In Experiment 1A, subjects practiced attending to shape and therefore had prior experience with shape information as relevant before probe trials. Drawing attention and behavioral responses to shape during practice could have activated competing rules as relevant throughout the prime. However, if mechanisms of attentional suppression are indeed shaped primarily by the experience of ignoring stimuli and maintaining their status across different contexts, then activation of competing rules and responses for shape information should not be necessary to observe a sustained attentional suppression. Experiment $1 \mathrm{~B}$ examined this issue by removing shape practice.

\section{Method}

Subjects. Seventy University of Toronto undergraduate students ( $\left.M_{\text {age }}=21.83, S D=2.4\right)$ participated for course credit or financial payment. Using the same prime performance criterion as in Experiment $1 \mathrm{~A}$, we removed three outliers from LI-two and one from LIfeature. Accordingly, each task contained the following number of subjects: baseline $(n=16)$, LI-one $(n=18)$, LI-two $(n=17)$, LI-feature $(n=15)$. 


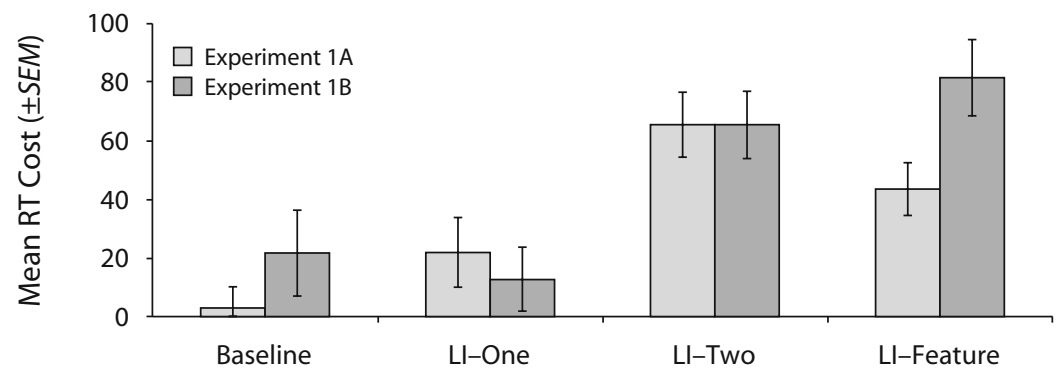

Figure 4. The mean reaction time $(\mathrm{RT})$ performance cost in milliseconds $( \pm S E M)$ across the entire probe block (i.e., collapsed across quartiles) for each task during Experiments 1A and 1B.

\section{Results}

In contrast to Experiment $1 \mathrm{~A}$, the accuracy performance cost during the probe block varied significantly as a function of task $[F(3,62)=4.156, p=.01]$. A significant accuracy decrement was observed during the probe for LI-two that was significantly greater than the baseline $[F(1,31)=12.14, p=.001, d=1.23]$ and LI-one costs $[F(1,33)=6.39, p=.016, d=0.88]$, and there was a trend toward a greater cost for LI-feature relative to baseline $[F(1,29)=3.10, p=.09, d=0.60]$.

Figure 4 depicts the mean RT cost, collapsed across quartiles, for each task in Experiments 1A and 1B. We replicated the RT results from Experiment 1A; the performance decrement during probe varied significantly as a function of task $[F(3,62)=6.935, p<.001]$. The LI-two cost was sustained and substantially greater than the baseline $[F(1,31)=5.59, p=.024, d=0.82]$ and LI-one $[F(1,33)=11.10, p=.002, d=1.11]$ costs. The LI-feature cost was similarly sustained and greater than the baseline cost $[F(1,29)=8.92, p=.006, d=1.07]$.

The findings from Experiment $1 \mathrm{~B}$ indicate that a sustained decrement during the probe can be observed in the absence of competing rules/responses for shape information, supporting the hypothesis that it is the experience of ignoring shape information that alters probe performance.

\section{DISCUSSION}

We found that when shape exemplars were ignored within a single-color-target context, they were transiently suppressed. In contrast, when the exemplars were consistently ignored for an equivalent number of trials across multiple-color-target contexts-hence, their task irrelevance was reinforced - the magnitude and duration of suppression increased and persisted throughout the entire 96-trial probe block. These data provide evidence that experience shapes attentional filters that suppress ignored information, with the crucial factor being whether stimuli are ignored consistently across different contexts, not just the amount of time they are ignored. Our findings build on a large body of behavioral (May, Kane, \& Hasher, 1995; Tipper, 1985, 2001), electrophysiological (e.g., Chelazzi, Miller, Duncan, \& Desimone, 1993; Reynolds \& Chelazzi,
2004), and neuroimaging (e.g., Kastner, De Weerd, Desimone, \& Ungerleider, 1998) work that has revealed an active suppression of ignored stimuli, including individual object features (e.g., Nobre, Rao, \& Chelazzi, 2006).

The data additionally revealed that with experience, suppression generalized beyond the specific shape exemplars ignored, to encompass shape processing in general (i.e., the feature level of shape). Both the baseline and LIfeature tasks involved responding to novel shapes after ignoring shape information for an equivalent amount of time. The baseline cost was negligible, whereas the LIfeature cost was robust and sustained, suggesting that the feature-based locus of suppression emerged only after the irrelevant status of the shape information was reinforced in that second-color context. Importantly, this suppression of the shape feature dimension does not reflect a general task-switch cost due to switching attention from color to shape, because it was measured as a decrement over and above the general shift cost assessed by our baseline task. These data demonstrate that the locus at which attentional suppression mechanisms operate adapts with experience.

It could be argued that the greater costs for LI-two and LI-feature were due to attending to two sets of color targets during the prime, as opposed to one, and that attention was therefore simply more attracted to color during the probe. However, this seems unlikely: (1) We specifically used novel colors during the probe block of all tasks, to minimize the influence of past color associations; (2) the greater cost for LI-one relative to baseline indicates that it was previous experience with shape information, and not color information alone, driving the probe performance - since they both involved attending to one set of color targets, but only the former involved reorienting attention to the previously ignored shapes. Furthermore, on the basis of the biased competition model of attention (Desimone \& Duncan, 1995), we assume that attention may have been more drawn to color information during the probe in LI-two and LI-feature, but this was as the result of our manipulation of subjects' experience of shape information throughout the prime blocks. This assumption is supported by the direct relationship between individuals' prime and probe performance when the ignored stimuli were presented in two-, rather than 
one-, color-target contexts, despite equal presentation of ignored stimuli. Thus, distractor stimuli are not simply ignored, and not processed, but rather, learning to ignore induces sustained attentional suppression and may serve as a fundamental mechanism by which behaviorally relevant stimuli are enhanced.

\section{AUTHOR NOTE}

We express gratitude to Asif Virani and Maryam Saheb-Al-Zamani for assistance in subject testing. This research was supported by NSERC and CFI/OIT grants awarded to E.D.R. Correspondence concerning this article should be addressed to E. De Rosa, Department of Psychology, University of Toronto, 100 Saint George Street, Toronto, ON M5S 3G3 Canada (e-mail: derosa@psych.utoronto.ca).

\section{REFERENCES}

Chelazzi, L., Miller, E. K., Duncan, J., \& Desimone, R. (1993). A neural basis for visual search in inferior temporal cortex. Nature, $\mathbf{3 6 3}$, 345-347. doi:10.1038/363345a0

Chun, M. M., \& JiAng, Y. (1998). Contextual cueing: Implicit learning and memory of visual context guides spatial attention. Cognitive Psychology, 36, 28-71. doi:10.1006/cogp.1998.0681

Chun, M. M., \& JiAng, Y. (1999). Top-down attentional guidance based on implicit learning of visual covariation. Psychological Science, 10, 360-365. doi:10.1111/1467-9280.00168

Desimone, R., \& Duncan, J. (1995). Neural mechanisms of selective visual attention. Annual Review of Neuroscience, 18, 193-222. doi:10.1146/annurev.ne.18.030195.001205

Dosher, B. A., \& Lu, Z. L. (1998). Perceptual learning reflects external noise filtering and internal noise reduction through channel reweighting. Proceedings of the National Academy of Sciences, 95, 1398813993. doi:10.1073/pnas.95.23.13988

FAHLE, M. (2005). Perceptual learning: Specificity versus generalization. Current Opinion in Neurobiology, 15, 154-160. doi:10.1016/j .conb.2005.03.010

ipata, A. E., Gee, A. L., Gottlieb, J., Bisley, J. W., \& Goldberg, M. E. (2006). LIP responses to a popout stimulus are reduced if it is overtly ignored. Nature Neuroscience, 9, 1071-1076. doi:10.1038/ nn1734
Kastner, S., De Weerd, P., Desimone, R., \& Ungerleider, L. G. (1998). Mechanisms of directed attention in the human extrastriate cortex as revealed by functional MRI. Science, 282, 108-111. doi:10.1126/science.282.5386.108

Mackintosh, N. J. (1975). A theory of attention: Variations in the associability of stimuli with reinforcement. Psychological Review, 82, 276-298. doi: 10.1037/h0076778

May, C. P., KANE, M. J., \& HASher, L. (1995). Determinants of negative priming. Psychological Bulletin, 118, 35-54.

NaVAlPaKKaM, V., \& ITti, L. (2007). Search goal tunes visual features optimally. Neuron, 53, 605-617. doi:10.1016/j.neuron.2007.01.018

Nobre, A. C., RaO, A., \& Chelazzi, L. (2006). Selective attention to specific features within objects: Behavioral and electrophysiological evidence. Journal of Cognitive Neuroscience, 18, 539-561. doi:10.1162/jocn.2006.18.4.539

Op de Beeck, H. P., Baker, C. I., DiCarlo, J. J., \& Kanwisher, N. G. (2006). Discrimination training alters object representations in human extrastriate cortex. Journal of Neuroscience, 26, 13025-13036. doi:10.1523/JNEUROSCI.2481-06.2006

Poggio, T., \& BizzI, E. (2004). Generalization in vision and motor control. Nature, 431, 768-774. doi:10.1038/nature03014

Reynolds, J. H., \& ChelazzI, L. (2004). Attentional modulation of visual processing. Annual Review of Neuroscience, 27, 611-647. doi:10.1146/annurev.neuro.26.041002.131039

Seitz, A. R., \& Watanabe, T. (2003). Psychophysics: Is subliminal learning really passive? Nature, 422, 36. doi:10.1038/422036a

Serences, J. T., \& Yantis, S. (2006). Selective visual attention and perceptual coherence. Trends in Cognitive Sciences, 10, 38-45. doi:10.1016/j.tics.2005.11.008

TIPPER, S. P. (1985). The negative priming effect: Inhibitory priming by ignored objects. Quarterly Journal of Experimental Psychology, 37A, 571-590. doi:10.1080/14640748508400920

TIPPER, S. P. (2001). Does negative priming reflect inhibitory mechanisms? A review and integration of conflicting views. Quarterly Journal of Experimental Psychology, 54A, 321-343. doi:10.1080/ 02724980042000183

Watanabe, T., NáÑez, J. E., \& SaSaki, Y. (2001). Perceptual learning without perception. Nature, 413, 844-848. doi:10.1038/35101601

(Manuscript received July 25, 2008; revision accepted for publication October 9, 2008.) 